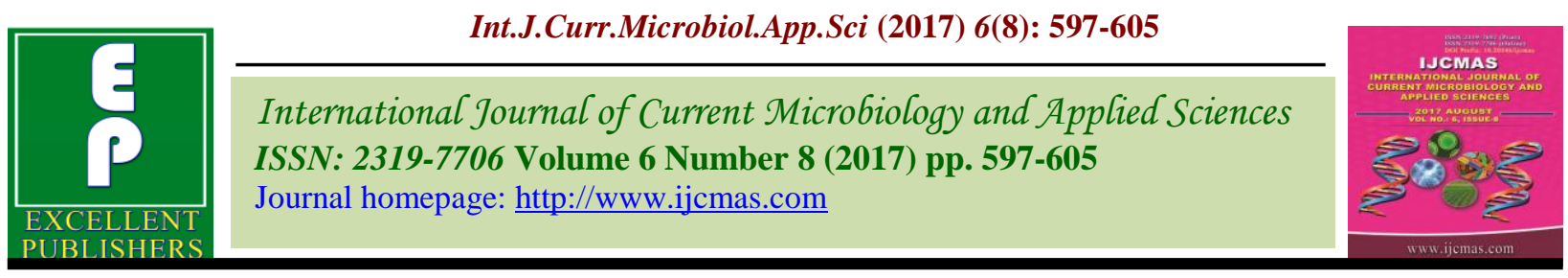

Original Research Article

https://doi.org/10.20546/ijcmas.2017.608.077

\title{
Evaluation of Coriander (Coriandrum sativum L.) Genotypes for Growth and Seed Yield Attributes
}

\author{
Akshay Jain", V.P. Pandey, V.B. Singh, Manvendra Singh and Sriom \\ Department of Vegetable Science Narendra Deva University of Agriculture and \\ Technology, Kumarganj, Faizabad-224229 (U.P.), India \\ *Corresponding author
}

\begin{tabular}{|c|c|}
\hline & A B S T R A C T \\
\hline & \multirow{6}{*}{ 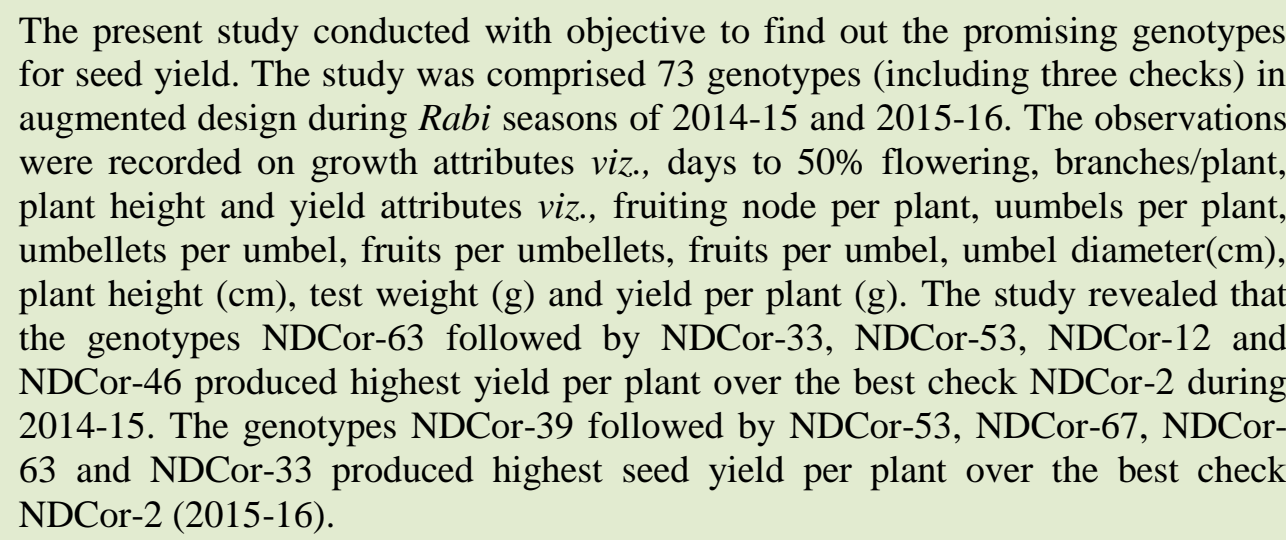 } \\
\hline Keywords & \\
\hline $\begin{array}{l}\text { Coriander, Seed } \\
\text { yield, Genotypes. }\end{array}$ & \\
\hline Article Info & \\
\hline $\begin{array}{l}\text { Accepted: } \\
\text { 04 June } 2017 \\
\text { Available Online: } \\
10 \text { August } 2017\end{array}$ & \\
\hline & \\
\hline
\end{tabular}

\section{Introduction}

The fascinating history of spices is a story of adventure exploration, congest and fierce naval rivalry. It was lure of these spices that brought many seafarers to the shore of India. It is the land of spices is the largest producer, consumer and exporter of spices in the world, where 63 kinds of spices are grown (Pruthi, 1998). Mainly 52 spices are grown in India according to Spices Board, Calicut, Kerala. Coriander is one of the important spices crop grown throughout the world.

It is a native of Mediterranean region. It is grown for both green leaves and dried seeds. In the country it is grown on an about 663 thousand hectare area with 609 million tonnes production and 1.0 million tonnes per hectare productivity and in Uttar Pradesh it is grown on an about 6.68 thousand hectare area with 2.61 million tonnes production and 2.6 tonnes per hectare productivity (Anonymous, 2015). It is extensively grown in Andhra Pradesh, Tamil Nadu, Karnataka, Punjab Rajasthan, Madhya Pradesh and Uttar Pradesh. Rajasthan is the major growing state and contributes about 40 per cent of coriander production in India Coriander (Coriandrum sativum L.) also called cilant or dhania is an annual herb, belong to the family Apiaceae. The genus Coriandrum comprised of two species. Among them, C. sativum is cultivated. The basic chromosome number of this genus is 
$\mathrm{x}=11$ and Corundum sativum is diploid with $2 n=22$. The plant is a smooth, erect annual herb, 60-100 cm high, with conspicuously enlarged nodes and hollow internodes. The stems are vertically ridged. The leaves are pinnately compound and often decompound. The lower leaves are broad with serratelylobed margins. The upper leaves are finely cut with linear lobes. The petiole is often swollen even, hollow at the base and sheathing the stem. The leaf arrangement is alternate. The plant comes to the flowering stage in about 45-60 days after sowing.

\section{Materials and Methods}

Experiments were conducted at the Main Experiment Station (Vegetable Research Farm), Narendra Deva University of Agriculture and Technology, (Narendra Nagar) Kumarganj, Faizabad (U.P.) India, in well leveled field having proper drainage. This farm is situated at Main Campus of the University on left side of Faizabad- Raebareli road at a distance of $42 \mathrm{~km}$ away from main city of Faizabad district. 73 lines including three checks of coriander germplasm maintained in All India Co-ordinated research project on spices under Department of Vegetable Science NDUAT, Kumarganj, Faizabad were taken for this investigation. These genotypes were collected from different places of India, The name of genotype and their source is given below table 1. The experiment was conducted in Augmented Block Design. The experiment consisting of 73 germplasm accessions was laid out in augmented block design.

\section{Results and Discussion}

\section{Days to 50\% flowering}

In $\mathrm{Y}_{1}$, the days to $50 \%$ flowering ranged from 84.67 (NDCor-22) to 94.67 days (NDCor-10) with the general mean of 89.42 days. However, the earliest 50\% flowering was recorded with the Pant Haritma among the three checks and in comparison to the best check i.e. Pant Haritma only five genotypes (NDCor-22, NDCor-5, NDCor-7, NDCor-8 and NDCor-29) took significantly lesser days to $50 \%$ flowering. In $\mathrm{Y}_{2}$, the days to $50 \%$ flowering ranged from 84.29 (NDCor-67) to 96.95 days (NDCor-37) with the general mean of 90.44 days. However, the earliest 50 $\%$ flowering was recorded with the Pant Haritma among the three checks and in comparison to the best check i.e. Pant Haritma seven genotypes (NDCor-67, NDCor-7, NDCor-15, NDCor-32, NDCor-50, NDCor-51, NDCor-66 and NDCor-67) took significantly lesser days to $50 \%$ flowering. This result is in close conformity to that of Islam et al., (2004).

\section{Branches per plant}

In $\mathrm{Y}_{1}$ number of branches per plant varied from 1.73 (NDCor-26) to 7.29 (NDCor-45) with the general mean of 4.47. Among the checks Pant Haritama recorded maximum (5.59) branches per plant. None of the Genotypes was found significantly superior to best check. The top five genotypes were NDCor-26, NDCor- 36, NDCor-17, NDCor67 and NDCor-9 for this trait. In $Y_{2}$ (2015-16) number of branches per plant varied from 3.43 (NDCor-4) to 9.62 (NDCor-59) with the general mean of 6.02. Among the checks Hissar Anand recorded maximum branches per plant. Fourteen genotypes were found significantly superior to best check. The top five genotypes were NDCor-59, NDCor- 12, NDCor-45, NDCor-6 and NDCor-56 for this trait. Rahman (2000) got branches per plant ranging from 6.10 to 8.02 .

\section{Fruiting nodes per plant}

In $\mathrm{Y}_{1}$ fruiting nodes per plant varied from 11.05 (NDCor-42) to 19.72 (NDCor-46) with the general mean of 15.17. Among the checks Pant Haritama recorded maximum (16.13) 
branches per plant. Nine Genotypes were found significantly superior to best check. The top five genotypes were NDCor-46, NDCor- 24 (18.33), NDCor-18 and (NDCor30 (18.15) and NDCor-35 (17.90) for this trait. In $\mathrm{Y}_{2}$ (2015-16) fruiting nodes per plant ranged from 11.77 (NDCor-61) to 17.56 (NDCor-36) with the general mean of 14.86 . Among the checks NDCor-2 recorded maximum branches per plant. Nineteen genotypes were found significantly superior to best check. The top five genotypes were NDCor-36, NDCor-32, NDCor-11, NDCor37 and NDCor-70 for this trait.

\section{Umbels per plant}

In $Y_{1}$ (2014-15) umbels per plant varied from 31.36 (NDCor-33) to 96.67 (NDCor-64) with the general mean of 68.71. Twenty nine genotypes were found significantly superior over the best check NDCor-2 for the trait umbels per plant. The top five genotypes in ranking were NDCor-64, NDCor-43, NDCor48, NDCor-62 and NDCor-65 (95.67) for this character. In $\mathrm{Y}_{2}$ (2015-16) umbels per plant varied from 30.24 (NDCor-40) to 88.48 (NDCor-50) with the general mean of 55.92. Twenty one genotypes were found significantly superior over the best check NDCor-2 for the trait umbels per plant. The top five genotypes in ranking were NDCor50, NDCor-54, NDCor-60, NDCor-51 and NDCor-59 for this character. But the findings about umbels per plant reported by Islam et al., (2004) and Anon., (2002b) were higher than the findings of the present study.

\section{Umbellets per umbel}

In $Y_{1}$ (2014-15) umbellets per umbel ranged from 4.90 (NDCor-4) to 8.90 (NDCor-20) with the general mean of 6.26. Only one genotype NDCor-20 (8.90) was found significantly superior over the best check Pant Haritma (7.13) among seventy genotypes, for this trait. In $\mathrm{Y}_{2}$ (2015-16) umbellets per umbel ranged from 4.93 (NDCor-4) to 8.23 (NDCor-69) with the general mean of 6.67. Only four genotypes viz., NDCor-69, NDCor61, NDCor-38 were found significantly superior over the best check NDCor-2 among seventy genotypes, for this trait. The result of this investigation corroborates the results of Maurya (1989).

\section{Fruits per umbellet}

In $\mathrm{Y}_{1}$ (2014-15) fruits per umbellet ranged from 5.53 (NDCor-8) to 11.55 (NDCor-58) with the general mean of 8.66. Twenty six genotypes were found significantly superior over the best check Hissar Anand (7.94) among seventy genotypes, for this trait. The top five ranked genotypes were NDCor-58 (11.55), NDCor-19, NDCor-20, NDCor-23 and NDCor-42. In $\mathrm{Y}_{2}(2015-16)$ fruits per umbellet ranged from 4.79 (NDCor-22) to 10.26 (NDCor-60) with the general mean of 7.10. Fourteen genotypes were found significantly superior over the best check Pant Haritma among seventy genotypes, for this trait. The top five ranked genotypes were NDCor-60, NDCor-42, NDCor-3, NDCor-55 and NDCor-64 (Table 2).

Table.1 Name and Source of genotypes studied in the experiment

\begin{tabular}{|c|l|l|}
\hline S. No. & Genotypes & Sources \\
\hline $\mathbf{1 .}$ & NDCor-1 & NDUA\&T, Kumarganj, Faizabad (U.P.) \\
\hline $\mathbf{2 .}$ & NDCor-3 & NDUA\&T, Kumarganj, Faizabad (U.P.) \\
\hline $\mathbf{3 .}$ & NDCor-4 & NDUA\&T, Kumarganj, Faizabad (U.P.) \\
\hline $\mathbf{4 .}$ & NDCor-5 & NDUA\&T, Kumarganj, Faizabad (U.P.) \\
\hline $\mathbf{5 .}$ & NDCor-6 & NDUA\&T, Kumarganj, Faizabad (U.P.) \\
\hline $\mathbf{6 .}$ & NDCor-7 & NDUA\&T, Kumarganj, Faizabad (U.P.) \\
\hline $\mathbf{7 .}$ & NDCor- 8 & NDUA\&T, Kumarganj, Faizabad (U.P.) \\
\hline $\mathbf{8 .}$ & NDCor- 9 & NDUA\&T, Kumarganj, Faizabad (U.P.) \\
\hline
\end{tabular}




\begin{tabular}{|c|c|c|}
\hline 9. & NDCor-10 & NDUA\&T, Kumarganj, Faizabad (U.P.) \\
\hline 10. & NDCor-11 & NDUA\&T, Kumarganj, Faizabad (U.P.) \\
\hline 11. & NDCor-12 & NDUA\&T, Kumarganj, Faizabad (U.P.) \\
\hline 12. & NDCor-13 & NDUA\&T, Kumarganj, Faizabad (U.P.) \\
\hline 13. & NDCor-14 & NDUA\&T, Kumarganj, Faizabad (U.P.) \\
\hline 14. & NDCor-15 & NDUA\&T, Kumarganj, Faizabad (U.P.) \\
\hline 15. & NDCor-16 & NDUA\&T, Kumarganj, Faizabad (U.P.) \\
\hline 16. & NDCor-17 & NDUA\&T, Kumarganj, Faizabad (U.P.) \\
\hline 17. & NDCor-18 & NDUA\&T, Kumarganj, Faizabad (U.P.) \\
\hline 18. & NDCor-19 & NDUA\&T, Kumarganj, Faizabad (U.P.) \\
\hline 19. & NDCor-20 & NDUA\&T, Kumarganj, Faizabad (U.P.) \\
\hline 20. & NDCor-21 & NDUA\&T, Kumarganj, Faizabad (U.P.) \\
\hline 21. & NDCor-22 & NDUA\&T, Kumarganj, Faizabad (U.P.) \\
\hline 22. & NDCor-23 & NDUA\&T, Kumarganj, Faizabad (U.P.) \\
\hline 23. & NDCor-24 & NDUA\&T, Kumarganj, Faizabad (U.P.) \\
\hline 24. & NDCor-25 & NDUA\&T, Kumarganj, Faizabad (U.P.) \\
\hline 25. & NDCor-26 & NDUA\&T, Kumarganj, Faizabad (U.P.) \\
\hline 26. & NDCor-27 & NDUA\&T, Kumarganj, Faizabad (U.P.) \\
\hline 27. & NDCor-28 & NDUA\&T, Kumarganj, Faizabad (U.P.) \\
\hline 28. & NDCor-29 & NDUA\&T, Kumarganj, Faizabad (U.P.) \\
\hline 29. & NDCor-30 & NDUA\&T, Kumarganj, Faizabad (U.P.) \\
\hline 30. & NDCor-31 & NDUA\&T, Kumarganj, Faizabad (U.P.) \\
\hline 31. & NDCor-32 & NDUA\&T, Kumarganj, Faizabad (U.P.) \\
\hline 32. & NDCor-33 & NDUA\&T, Kumarganj, Faizabad (U.P.) \\
\hline 33. & NDCor-34 & NDUA\&T, Kumarganj, Faizabad (U.P.) \\
\hline 34. & NDCor-35 & NDUA\&T, Kumarganj, Faizabad (U.P.) \\
\hline 35. & NDCor-36 & NDUA\&T, Kumarganj, Faizabad (U.P.) \\
\hline 36. & NDCor-37 & NDUA\&T, Kumarganj, Faizabad (U.P.) \\
\hline 37. & NDCor-38 & NDUA\&T, Kumarganj, Faizabad (U.P.) \\
\hline 38. & NDCor-39 & NDUA\&T, Kumarganj, Faizabad (U.P.) \\
\hline 39. & NDCor-40 & NDUA\&T, Kumarganj, Faizabad (U.P.) \\
\hline 40. & NDCor-41 & NDUA\&T, Kumarganj, Faizabad (U.P.) \\
\hline 41. & NDCor-42 & NDUA\&T, Kumarganj, Faizabad (U.P.) \\
\hline 42. & NDCor-43 & NDUA\&T, Kumarganj, Faizabad (U.P.) \\
\hline 43. & NDCor-44 & NDUA\&T, Kumarganj, Faizabad (U.P.) \\
\hline 44. & NDCor-45 & NDUA\&T, Kumarganj, Faizabad (U.P.) \\
\hline 45. & NDCor-46 & NDUA\&T, Kumarganj, Faizabad (U.P.) \\
\hline 46. & NDCor-47 & NDUA\&T, Kumarganj, Faizabad (U.P.) \\
\hline 47. & NDCor-48 & NDUA\&T, Kumarganj, Faizabad (U.P.) \\
\hline 48. & NDCor-49 & NDUA\&T, Kumarganj, Faizabad (U.P.) \\
\hline 49. & NDCor-50 & NDUA\&T, Kumarganj, Faizabad (U.P.) \\
\hline 50. & NDCor-51 & NDUA\&T, Kumarganj, Faizabad (U.P.) \\
\hline 51. & NDCor-52 & NDUA\&T, Kumarganj, Faizabad (U.P.) \\
\hline 52. & NDCor-53 & NDUA\&T, Kumarganj, Faizabad (U.P.) \\
\hline 53. & NDCor-54 & NDUA\&T, Kumarganj, Faizabad (U.P.) \\
\hline 54. & NDCor-55 & NDUA\&T, Kumarganj, Faizabad (U.P.) \\
\hline 55. & NDCor-56 & NDUA\&T, Kumarganj, Faizabad (U.P.) \\
\hline 56. & NDCor-57 & NDUA\&T, Kumarganj, Faizabad (U.P.) \\
\hline 57. & NDCor-58 & NDUA\&T, Kumarganj, Faizabad (U.P.) \\
\hline 58. & NDCor-59 & NDUA\&T, Kumarganj, Faizabad (U.P.) \\
\hline 59. & NDCor-60 & NDUA\&T, Kumarganj, Faizabad (U.P.) \\
\hline 60. & NDCor-61 & NDUA\&T, Kumarganj, Faizabad (U.P.) \\
\hline 61. & NDCor-62 & NDUA\&T, Kumarganj, Faizabad (U.P.) \\
\hline 62. & NDCor-63 & NDUA\&T, Kumarganj, Faizabad (U.P.) \\
\hline 63. & NDCor-64 & NDUA\&T, Kumarganj, Faizabad (U.P.) \\
\hline 64. & NDCor-65 & NDUA\&T, Kumarganj, Faizabad (U.P.) \\
\hline 65. & NDCor-66 & NDUA\&T, Kumarganj, Faizabad (U.P.) \\
\hline 66. & NDCor-67 & NDUA\&T, Kumarganj, Faizabad (U.P.) \\
\hline 67. & NDCor-68 & NDUA\&T, Kumarganj, Faizabad (U.P.) \\
\hline 68. & NDCor-69 & NDUA\&T, Kumarganj, Faizabad (U.P.) \\
\hline 69. & NDCor-70 & NDUA\&T, Kumarganj, Faizabad (U.P.) \\
\hline 70. & NDCor-71 & NDUA\&T, Kumarganj, Faizabad (U.P.) \\
\hline 71. & Pant Haritma $(\mathrm{Ch})$ & GBPUAT, Pantnagar, Uttrakhand \\
\hline 72. & Hisar Aanand (Ch) & CCS Haryana Agriculture University, Hisar (Haryana) \\
\hline 73. & NDCor-2 (Ch) & NDUA\&T, Kumarganj, Faizabad (U.P.) \\
\hline
\end{tabular}


Table.2 Mean performance of genotypes during Rabi seasons 2014-15 and 2015-16

\begin{tabular}{|c|c|c|c|c|c|c|c|c|c|c|c|c|c|c|c|c|c|c|c|c|c|c|c|}
\hline \multirow[t]{2}{*}{$\begin{array}{l}\text { S. } \\
\text { No } \\
\text {. }\end{array}$} & \multirow{2}{*}{$\begin{array}{l}\text { Characters } \\
\text { Genotypes }\end{array}$} & \multicolumn{2}{|c|}{$\begin{array}{l}\text { Days to } 50 \% \\
\text { flowering }\end{array}$} & \multicolumn{2}{|c|}{$\begin{array}{c}\text { Branches/ } \\
\text { plant }\end{array}$} & \multicolumn{2}{|c|}{$\begin{array}{c}\text { Fruiting nodes/ } \\
\text { plant }\end{array}$} & \multicolumn{2}{|c|}{ Umbels / plant } & \multicolumn{2}{|c|}{$\begin{array}{c}\text { Umbellets/ } \\
\text { umbel }\end{array}$} & \multicolumn{2}{|c|}{$\begin{array}{c}\text { Fruits/ } \\
\text { umbellet }\end{array}$} & \multicolumn{2}{|c|}{ Fruits/ umbel } & \multicolumn{2}{|c|}{$\begin{array}{c}\text { Umbel } \\
\text { diameter } \\
(\mathrm{cm})\end{array}$} & \multicolumn{2}{|c|}{ Plant height $(\mathbf{c m})$} & \multicolumn{2}{|c|}{ Test weight (g) } & \multicolumn{2}{|c|}{ Yield/ plant (g) } \\
\hline & & $\mathbf{Y}_{1}$ & $\mathbf{Y}_{2}$ & $\mathbf{Y}_{1}$ & $\mathbf{Y}_{2}$ & $\mathbf{Y}_{1}$ & $\mathbf{Y}_{2}$ & $\mathbf{Y}_{1}$ & $\mathbf{Y}_{2}$ & $Y_{1}$ & $\mathbf{Y}_{2}$ & $\mathbf{Y}_{1}$ & $\mathbf{Y}_{2}$ & $\mathbf{Y}_{1}$ & $\mathbf{Y}_{2}$ & $\mathbf{Y}_{1}$ & $\mathbf{Y}_{2}$ & $\mathbf{Y}_{1}$ & $\mathbf{Y}_{2}$ & $\mathbf{Y}_{1}$ & $\mathbf{Y}_{2}$ & $\mathbf{Y}_{1}$ & $\mathbf{Y}_{2}$ \\
\hline 1 & NDCor-1 & 89.67 & 88.29 & 4.10 & 6.61 & 11.84 & 14.37 & 61.30 & 58.94 & 4.90 & 6.90 & 10.73 & 6.79 & 37.74 & 36.09 & 5.86 & 5.40 & 127.06 & 134.14 & 8.91 & 10.69 & 11.60 & 10.15 \\
\hline 2 & NDCor-3 & 86.67 & 90.29 & 6.30 & 3.86 & 11.54 & 12.76 & 88.50 & 46.14 & 6.30 & 6.70 & 8.53 & 9.59 & 34.54 & 45.49 & 4.46 & 6.17 & 142.46 & 145.79 & 8.25 & 9.57 & 14.03 & 14.27 \\
\hline 3 & NDCor-4 & 88.67 & 92.29 & 3.30 & 3.43 & 13.39 & 15.38 & 60.10 & 35.54 & 5.70 & 7.00 & 6.03 & 6.79 & 34.94 & 51.09 & 6.06 & 6.02 & 133.10 & 140.81 & 9.03 & 10.49 & 16.97 & 17.42 \\
\hline 4 & NDCor-5 & 85.67 & 94.29 & 2.10 & 6.21 & 11.60 & 16.57 & 41.30 & 65.64 & 6.30 & 6.90 & 8.93 & 6.69 & 36.54 & 45.79 & 6.66 & 5.25 & 133.12 & 149.99 & 11.01 & 9.76 & 17.09 & 16.34 \\
\hline 5 & NDCor-6 & 88.67 & 93.29 & 3.50 & 8.32 & 11.73 & 13.01 & 56.50 & 51.34 & 7.30 & 6.80 & 9.73 & 6.39 & 36.34 & 32.89 & 6.26 & 5.70 & 140.94 & 137.21 & 11.41 & 9.71 & 12.44 & 9.63 \\
\hline 6 & NDCor-7 & 85.67 & 87.29 & 3.75 & 7.61 & 14.32 & 15.59 & 63.50 & 66.94 & 5.70 & 6.10 & 10.53 & 5.29 & 37.54 & 43.39 & 5.06 & 6.54 & 132.36 & 144.39 & 8.31 & 9.48 & 13.10 & 9.92 \\
\hline 7 & NDCor-8 & 85.67 & 90.29 & 3.52 & 6.60 & 15.53 & 13.28 & 46.50 & 40.34 & 5.10 & 7.50 & 5.53 & 8.09 & 38.34 & 46.89 & 6.86 & 6.20 & 121.86 & $\mathbf{1 3 7 . 2 3}$ & 9.11 & 9.99 & 11.10 & 7.85 \\
\hline 8 & NDCor-9 & 90.67 & 92.29 & 6.75 & 6.01 & 16.54 & 12.77 & $\mathbf{5 3 . 5 0}$ & 44.54 & 7.10 & 6.40 & 7.13 & 6.39 & 36.14 & 28.99 & 5.06 & 4.95 & 143.46 & 148.15 & 11.41 & 11.89 & 9.33 & 12.35 \\
\hline 9 & NDCor-10 & 94.67 & 93.29 & 4.84 & 5.22 & 14.33 & 13.35 & 57.90 & 56.94 & 5.30 & 6.90 & 8.13 & 6.49 & 37.34 & 46.29 & 6.66 & 6.00 & 147.76 & 134.24 & 11.45 & 9.50 & 16.41 & 15.53 \\
\hline 10 & NDCor-11 & 89.67 & 94.29 & 4.78 & 4.65 & 15.84 & 16.89 & 55.50 & 50.94 & 6.50 & 6.30 & 5.93 & 5.39 & 34.94 & 29.09 & 6.26 & 6.65 & 140.60 & 142.21 & 10.85 & 9.66 & 11.66 & 13.28 \\
\hline 11 & NDCor-12 & 90.67 & 95.95 & 4.42 & 9.16 & 15.33 & 14.54 & 53.17 & 67.14 & 5.90 & 6.23 & 10.68 & 7.19 & 36.90 & 48.79 & 5.70 & 6.57 & 122.43 & 144.46 & 10.06 & 13.98 & 17.31 & 17.02 \\
\hline 12 & NDCor-13 & 91.67 & 96.95 & 2.82 & 6.26 & 14.75 & 13.93 & 31.57 & 65.34 & 5.50 & 7.03 & 6.08 & 8.59 & 39.30 & 44.99 & 5.30 & 5.08 & 124.71 & 141.14 & 10.35 & 13.79 & 16.73 & 16.35 \\
\hline 13 & NDCor-14 & 86.67 & 95.95 & 4.69 & 5.46 & 15.31 & 14.23 & 46.17 & 45.84 & 5.10 & 5.63 & 7.28 & 7.49 & 32.70 & 34.39 & 7.00 & 6.65 & 138.23 & 129.02 & 10.52 & 12.51 & 12.40 & 15.28 \\
\hline 14 & NDCor15 & 87.67 & 85.95 & 3.65 & 5.96 & 13.59 & 16.54 & 65.57 & 50.44 & 5.90 & 6.03 & 6.68 & 6.59 & 35.90 & 46.99 & 7.30 & 5.67 & 122.63 & 149.55 & 9.65 & 12.99 & 10.83 & 12.78 \\
\hline 15 & NDCor-16 & 89.67 & 89.95 & 6.22 & 6.76 & 16.18 & 12.66 & 76.17 & 65.64 & 6.50 & 7.53 & 10.08 & 7.39 & 35.30 & 45.89 & 5.30 & 7.16 & 136.63 & 148.48 & 8.95 & 11.30 & 13.40 & 13.50 \\
\hline 16 & NDCor-17 & 89.67 & 90.95 & 6.86 & 6.66 & 15.15 & 13.94 & 69.97 & 61.04 & 7.50 & 6.63 & 7.08 & 5.79 & 35.70 & 33.29 & 4.60 & 6.52 & 123.43 & 146.61 & 9.50 & 11.99 & 14.91 & 16.02 \\
\hline 17 & NDCor-18 & 93.67 & 89.95 & 3.62 & 7.16 & 18.15 & 13.12 & 72.77 & 59.44 & 5.70 & 6.23 & 8.88 & 5.59 & 40.90 & 32.99 & 4.40 & 5.95 & 128.03 & 116.52 & 9.70 & 10.84 & 12.40 & 9.64 \\
\hline 18 & NDCor-19 & 89.67 & 92.95 & 3.82 & 4.96 & 17.13 & 14.14 & 73.97 & 61.64 & 5.90 & 6.83 & 11.30 & 5.79 & 34.50 & 41.79 & 3.60 & 6.00 & 126.57 & 130.53 & 8.50 & 9.11 & 11.60 & 12.38 \\
\hline 19 & NDCor-20 & 91.67 & 88.95 & 6.19 & 4.56 & 15.73 & 15.09 & 53.97 & 72.84 & 8.90 & 6.23 & 11.08 & 5.69 & 36.30 & 41.39 & 4.90 & 5.28 & 136.43 & 126.54 & 11.15 & 11.71 & 14.51 & 15.25 \\
\hline 20 & NDCor-21 & 90.67 & 89.95 & 3.02 & 6.76 & 14.83 & 15.74 & 33.37 & 68.24 & 5.70 & 6.33 & 6.48 & 4.79 & 38.70 & 31.19 & 5.50 & 5.50 & 110.39 & 128.55 & 8.80 & 13.99 & 13.50 & 8.98 \\
\hline 21 & NDCor-22 & 84.67 & 89.95 & 2.53 & 6.26 & 16.33 & 13.43 & 69.97 & 71.48 & 7.44 & 6.03 & 8.88 & 5.86 & 39.40 & 41.85 & 5.33 & 6.49 & 139.63 & 140.27 & 11.12 & 11.13 & 14.92 & 11.09 \\
\hline 22 & NDCor-23 & 86.67 & 88.95 & 4.00 & 5.06 & 17.33 & 15.24 & 75.37 & 62.98 & 6.44 & 5.83 & 11.08 & 5.46 & 40.20 & 29.45 & 7.13 & 6.38 & 134.67 & 147.29 & 10.60 & 14.04 & 16.56 & 17.39 \\
\hline 23 & NDCor-24 & 88.67 & 89.95 & 5.75 & 5.16 & 18.33 & 15.45 & 62.17 & 59.58 & 6.34 & 7.73 & 7.88 & 5.86 & 38.40 & 47.65 & 5.23 & 6.53 & 160.83 & 147.25 & 10.75 & 12.15 & 11.61 & 8.26 \\
\hline 24 & NDCor-25 & 86.67 & 93.95 & 2.99 & 4.46 & 16.33 & 16.24 & 66.57 & 60.18 & 6.84 & 5.23 & 8.08 & 5.46 & 33.60 & 34.35 & 4.43 & 6.80 & 140.03 & 140.25 & 10.58 & 9.60 & 13.32 & 9.23 \\
\hline 25 & NDCor-26 & 87.67 & 89.95 & 1.73 & 5.86 & 16.02 & 15.87 & 48.57 & 72.58 & 6.24 & 5.83 & 10.48 & 6.26 & 36.60 & 36.25 & 4.13 & 5.84 & 127.13 & 146.19 & 10.55 & 13.16 & 16.32 & 17.00 \\
\hline 26 & NDCor-27 & 93.67 & 88.95 & 2.77 & 7.06 & 16.12 & 16.02 & 59.77 & 60.78 & 6.44 & 5.63 & 8.68 & 5.46 & 37.60 & 30.05 & 4.03 & 6.51 & 118.35 & 142.21 & 9.80 & 12.15 & 13.20 & 10.69 \\
\hline 27 & NDCor-28 & 88.67 & 89.95 & 4.21 & 6.46 & 17.15 & 15.84 & 63.77 & 56.18 & 5.64 & 6.23 & 8.68 & 5.46 & 37.40 & 40.05 & 4.73 & 6.24 & 118.99 & 147.23 & 9.84 & 8.16 & 16.28 & 16.39 \\
\hline
\end{tabular}


Contd.........

\begin{tabular}{|c|c|c|c|c|c|c|c|c|c|c|c|c|c|c|c|c|c|c|c|c|c|c|c|}
\hline \multirow{2}{*}{$\begin{array}{l}\text { S. } \\
\mathbf{N} \\
\mathbf{o .}\end{array}$} & \multirow[t]{2}{*}{$\begin{array}{c}\text { Characters } \\
\text { Genotypes }\end{array}$} & \multicolumn{2}{|c|}{$\begin{array}{l}\text { Days to } 50 \% \\
\text { flowering }\end{array}$} & \multicolumn{2}{|c|}{$\begin{array}{c}\text { Branches/ } \\
\text { plant }\end{array}$} & \multicolumn{2}{|c|}{$\begin{array}{c}\text { Fruiting nodes/ } \\
\text { plant }\end{array}$} & \multicolumn{2}{|c|}{ Umbels / plant } & \multicolumn{2}{|c|}{$\begin{array}{c}\text { Umbellets/ } \\
\text { umbel }\end{array}$} & \multicolumn{2}{|c|}{$\begin{array}{c}\text { Fruits/ } \\
\text { umbellet }\end{array}$} & \multicolumn{2}{|c|}{ Fruits/ umbel } & \multicolumn{2}{|c|}{$\begin{array}{c}\text { Umbel } \\
\text { diameter } \\
(\mathbf{c m})\end{array}$} & \multicolumn{2}{|c|}{ Plant height $(\mathbf{c m})$} & \multicolumn{2}{|c|}{ Test weight (g) } & \multicolumn{2}{|c|}{ Yield/ plant (g) } \\
\hline & & $\mathrm{Y}_{1}$ & $\mathrm{Y}_{2}$ & $\mathrm{Y}_{1}$ & $\mathrm{Y}_{2}$ & $\mathrm{Y}_{1}$ & $\mathrm{Y}_{2}$ & $\mathrm{Y}_{1}$ & $\mathrm{Y}_{2}$ & $\mathrm{Y}_{1}$ & $\mathrm{Y}_{2}$ & $\mathrm{Y}_{1}$ & $\mathrm{Y}_{2}$ & $\mathrm{Y}_{1}$ & $\mathrm{Y}_{2}$ & $\mathrm{Y}_{1}$ & $\mathrm{Y}_{2}$ & $\mathrm{Y}_{1}$ & $\mathrm{Y}_{2}$ & $\mathrm{Y}_{1}$ & $\mathrm{Y}_{2}$ & $\mathrm{Y}_{1}$ & $\mathrm{Y}_{2}$ \\
\hline 28. & NDCor-29 & 85.67 & 87.95 & 3.93 & 5.06 & 17.05 & 15.49 & 60.57 & 60.68 & 6.44 & 6.13 & 9.28 & 6.86 & 38.30 & 39.45 & 3.23 & 5.44 & 129.03 & 135.24 & 10.77 & 12.18 & 14.46 & 13.24 \\
\hline 29. & NDCor-30 & 90.67 & 88.95 & 4.08 & 5.76 & 18.15 & 15.13 & 73.57 & 46.98 & 5.84 & 6.43 & 9.28 & 5.86 & 35.80 & 41.75 & 4.03 & 6.94 & 120.03 & 144.19 & 10.53 & 12.05 & 12.20 & 11.11 \\
\hline 30. & NDCor-31 & 88.67 & 86.95 & 3.53 & 6.26 & 17.22 & 15.44 & 69.57 & 51.98 & 7.54 & 6.03 & 7.68 & 7.46 & 39.50 & 52.45 & 4.03 & 5.76 & 121.83 & 135.25 & 10.80 & 9.56 & 16.12 & 16.79 \\
\hline 31. & NDCor-32 & 89.67 & 87.29 & 4.98 & 5.99 & 15.26 & 17.52 & 61.16 & 63.94 & 5.47 & 4.93 & 8.41 & 5.96 & 35.87 & 32.12 & 4.03 & 5.41 & 113.74 & 137.86 & 11.12 & 9.95 & 11.35 & 10.49 \\
\hline 32. & NDCor-33 & 87.67 & 89.29 & 4.28 & 7.39 & 12.87 & 16.14 & 31.36 & 50.84 & 6.67 & 5.83 & 7.21 & 5.56 & 37.47 & 31.92 & 4.83 & 4.94 & 125.14 & 144.82 & 11.02 & 11.32 & 17.43 & 17.81 \\
\hline 33. & NDCor-34 & 93.67 & 90.29 & 5.26 & 4.79 & 16.47 & 16.25 & 72.36 & 43.64 & 5.67 & 5.53 & 8.61 & 6.26 & 36.67 & 31.72 & 4.03 & 7.65 & 138.74 & 145.78 & 12.41 & 9.54 & 9.71 & 6.89 \\
\hline 34. & NDCor-35 & 91.67 & 90.29 & 6.37 & 5.99 & 17.90 & 16.33 & 38.16 & 47.54 & 6.47 & 6.43 & 8.01 & 6.16 & 38.67 & 40.92 & 3.93 & 7.66 & 121.94 & 140.82 & 9.15 & 12.15 & 11.40 & 9.75 \\
\hline 35 . & NDCor-36 & 88.67 & 91.29 & 6.92 & 5.19 & 16.80 & 17.56 & 70.76 & 35.84 & 5.67 & 5.33 & 9.01 & 5.36 & 38.87 & 31.72 & 4.23 & 6.74 & 128.14 & 135.78 & 9.36 & 9.86 & 12.79 & 10.83 \\
\hline 36. & NDCor-37 & 86.67 & 95.29 & 4.78 & 5.39 & 15.90 & 16.93 & 78.16 & 46.64 & 7.47 & 6.53 & 9.61 & 6.56 & 38.47 & 44.72 & 4.43 & 6.57 & 140.14 & 137.80 & 10.87 & 10.35 & 9.10 & 7.14 \\
\hline 37. & NDCor-38 & 89.67 & 91.29 & 6.28 & 4.49 & 15.87 & 14.97 & 81.16 & 31.84 & 6.67 & 7.93 & 10.21 & 7.86 & 37.47 & 50.72 & 4.93 & 5.54 & 133.14 & 126.01 & 10.71 & 8.10 & 10.02 & 9.90 \\
\hline 38. & NDCor-39 & 86.67 & 88.29 & 5.18 & 5.29 & 14.77 & 15.33 & 55.96 & 31.44 & 6.47 & 6.13 & 11.01 & 6.56 & 39.47 & 38.12 & 6.83 & 6.13 & 128.74 & 129.80 & 11.32 & 9.65 & 16.57 & 18.99 \\
\hline 39. & NDCor-40 & 90.67 & 89.29 & 4.18 & 5.19 & 16.87 & 15.28 & 87.76 & 30.24 & 6.07 & 6.23 & 10.01 & 6.46 & 36.87 & 40.52 & 5.63 & 6.09 & 139.74 & 116.38 & 11.30 & 11.17 & 14.38 & 17.17 \\
\hline 40. & NDCor-41 & 92.67 & 90.29 & 5.16 & 5.79 & 12.00 & 14.79 & 81.76 & 34.84 & 6.87 & 5.73 & 9.41 & 7.36 & 37.47 & 40.32 & 3.93 & 5.66 & 134.94 & 127.32 & 9.15 & 9.35 & 14.70 & 15.96 \\
\hline 41. & NDCor-42 & 91.33 & 87.62 & 3.67 & 5.71 & 11.05 & 15.93 & 83.28 & 49.58 & 6.54 & 7.67 & 11.08 & 9.66 & 38.77 & 52.72 & 4.13 & 5.88 & 140.42 & 142.17 & 10.52 & 9.75 & 12.42 & 9.76 \\
\hline 42. & NDCor-43 & 90.33 & 85.62 & 6.03 & 7.98 & 14.65 & 13.93 & 92.08 & 58.88 & 7.14 & 6.57 & 9.38 & 7.06 & 38.47 & 38.62 & 3.93 & 6.09 & 137.62 & 158.28 & 9.71 & 9.70 & 10.49 & 9.50 \\
\hline 43. & NDCor-44 & 92.33 & 89.62 & 3.47 & 8.61 & 15.01 & 16.33 & 87.68 & 58.98 & 6.24 & 6.67 & 7.78 & 7.46 & 39.77 & 47.22 & 4.83 & 5.52 & 129.82 & 149.59 & 11.45 & 13.14 & 16.29 & 17.56 \\
\hline 44. & NDCor-45 & 90.33 & 94.62 & 7.29 & 9.01 & 17.72 & 15.38 & 71.28 & 56.98 & 6.54 & 6.87 & 9.58 & 8.86 & 36.37 & 47.32 & 4.63 & 6.72 & 129.22 & 134.80 & 10.68 & 9.75 & 9.50 & 8.11 \\
\hline 45. & NDCor-46 & & & & 6.05 & & & 54.28 & & 6.34 & 6.47 & & & & & & & & & & 9.75 & & 17.64 \\
\hline 46. & NDCor- & 86.33 & 90.62 & 4.87 & 5.37 & 14.83 & 14.71 & 77.28 & 58 & $\frac{0.04}{5.44}$ & $\begin{array}{l}7.47 \\
7.07\end{array}$ & 9.98 & 5.66 & $\begin{array}{l}36.27 \\
6.9\end{array}$ & 36.92 & 4.03 & 6.73 & $\begin{array}{l}1.30 .02 \\
124.35\end{array}$ & 140 & 9.32 & 9.54 & 9.48 & 6.27 \\
\hline 47. & NDCor-48 & 88.33 & 88.62 & 6.07 & 7.41 & 15.05 & 13.53 & 90.08 & 46.58 & 6.14 & 6.07 & 8.48 & 7.26 & 34.97 & 43.72 & 6.93 & 6.44 & 119.22 & 140.43 & 9.29 & 9.46 & 11.76 & 10.56 \\
\hline 48. & NDCor-49 & 90.33 & 86.62 & 4.27 & 5.52 & 16.22 & 15.58 & 87.88 & 62.78 & 5.54 & 6.87 & 6.18 & 7.66 & 38.37 & 49.52 & 4.13 & 6.42 & 105.79 & 138.64 & 10.12 & 9.57 & 13.82 & 15.15 \\
\hline 49. & NDCor-50 & 89.33 & 84.62 & 4.94 & 5.81 & 11.72 & 15.93 & 50.48 & 88.48 & 5.74 & 7.37 & 9.98 & 8.06 & 37.07 & 67.12 & 4.73 & 6.18 & 96.62 & 146.77 & 8.97 & 8.74 & 14.20 & 14.94 \\
\hline 50. & NDCor-51 & 90.33 & 85.62 & 4.47 & 7.61 & 13.32 & 16.33 & 71.08 & 78.98 & 6.34 & 6.97 & 7.98 & 7.46 & 36.97 & 48.52 & 2.43 & 6.49 & 111.22 & 142.65 & 9.91 & 9.25 & 13.59 & 14.45 \\
\hline 51. & NDCor-52 & 91.00 & 90.62 & 3.57 & 5.55 & 15.74 & 13.86 & 73.86 & 43.34 & 5.50 & 6.40 & 6.75 & 7.76 & 37.87 & 41.05 & 5.20 & 5.25 & 120.31 & 115.04 & 9.43 & 10.46 & 12.89 & 10.69 \\
\hline 52. & NDCor-53 & 87.00 & 91.62 & 3.17 & 3.97 & 17.68 & 12.64 & 80.66 & 36.14 & 6.30 & 7.10 & 9.95 & 7.66 & 36.27 & 56.45 & 5.10 & 5.30 & 124.91 & 125.23 & 10.29 & 10.57 & 17.32 & 18.42 \\
\hline 53. & NDCor-54 & 89.00 & 92.62 & 3.37 & 6.62 & 14.69 & 13.65 & 86.46 & 83.74 & 6.20 & 7.00 & 7.35 & 7.46 & 34.87 & 43.65 & 6.20 & 5.38 & 129.54 & 146.03 & 10.54 & 9.55 & 13.96 & 16.88 \\
\hline 54. & NDCor-55 & 86.00 & 93.62 & 5.11 & 5.02 & 14.09 & 14.78 & 80.40 & 48.24 & $\frac{0.20}{5.10}$ & 7.20 & 6.95 & 9.26 & 37.17 & 59.25 & 6.00 & 5.63 & 130.11 & 138.67 & 8.78 & 12.50 & 10.58 & 8.57 \\
\hline
\end{tabular}

Contd.........

\begin{tabular}{|c|c|c|c|c|c|c|c|c|c|c|c|c|c|c|c|c|c|c|c|c|c|c|c|}
\hline \multirow[t]{2}{*}{$\begin{array}{l}\text { S. } \\
\text { No }\end{array}$} & \multirow{2}{*}{ Genotypes } & \multicolumn{2}{|c|}{$\begin{array}{l}\text { Days to 50\% } \\
\text { flowering }\end{array}$} & \multicolumn{2}{|c|}{ Branches/ plant } & \multicolumn{2}{|c|}{$\begin{array}{c}\text { Fruiting nodes/ } \\
\text { plant }\end{array}$} & \multicolumn{2}{|c|}{ Umbels / plant } & \multicolumn{2}{|c|}{ Umbellets/ umbel } & \multicolumn{2}{|c|}{ Fruits/ umbellet } & \multicolumn{2}{|c|}{ Fruits/ umbel } & \multicolumn{2}{|c|}{$\begin{array}{c}\text { Umbel } \\
\text { diameter }(\mathrm{cm})\end{array}$} & \multicolumn{2}{|c|}{ Plant height (cm) } & \multicolumn{2}{|c|}{ Test weight (g) } & \multicolumn{2}{|c|}{ Yield/ plant (g) } \\
\hline & & $\mathbf{Y}_{1}$ & $\mathbf{Y}_{2}$ & $\mathbf{Y}_{1}$ & $\mathbf{Y}_{2}$ & $\mathbf{Y}_{1}$ & $\mathbf{Y}_{2}$ & $\mathbf{Y}_{1}$ & $\mathbf{Y}_{2}$ & $\mathbf{Y}_{1}$ & $\mathbf{Y}_{2}$ & $\mathbf{Y}_{1}$ & $\mathbf{Y}_{2}$ & $\mathbf{Y}_{1}$ & $\mathbf{Y}_{2}$ & $\mathbf{Y}_{1}$ & $\mathbf{Y}_{2}$ & $\mathbf{Y}_{1}$ & $\mathbf{Y}_{2}$ & $\mathbf{Y}_{1}$ & $\mathbf{Y}_{2}$ & $\mathbf{Y}_{1}$ & $\mathbf{Y}_{2}$ \\
\hline 55 & NDCor-56 & 94.00 & 89.62 & 3.77 & 8.16 & 12.87 & 15.74 & 78.46 & 76.14 & 5.50 & 7.60 & 7.55 & 8.36 & 38.07 & 52.15 & 6.10 & 6.13 & 122.11 & 128.05 & 9.69 & 10.56 & 10.96 & 13.44 \\
\hline 56 & NDCor-57 & 90.00 & 90.62 & 6.56 & 5.55 & 11.85 & 13.17 & 78.86 & 74.54 & 6.40 & 6.70 & 9.95 & 6.46 & 39.57 & 43.25 & 5.90 & 6.15 & 128.91 & 124.03 & 9.53 & 11.59 & 15.32 & 12.23 \\
\hline 57 & NDCor-58 & 92.00 & 92.62 & 2.97 & 6.38 & 13.14 & 12.59 & 81.66 & 68.14 & 5.70 & 6.40 & 11.55 & 6.86 & 37.07 & 43.55 & 7.20 & 4.98 & 132.71 & 118.23 & 10.31 & 11.46 & 13.88 & 15.18 \\
\hline 58 & NDCor-59 & 86.00 & 93.62 & 4.50 & 9.62 & 14.76 & 13.81 & 85.46 & 77.94 & 6.20 & 7.90 & 9.75 & 6.56 & 37.47 & 44.05 & 7.30 & 5.77 & 113.85 & 133.50 & 11.17 & 11.57 & 12.89 & 12.68 \\
\hline 59 & NDCor-60 & 93.00 & 95.62 & 3.97 & 6.21 & 15.25 & 12.64 & 47.86 & 81.64 & 7.00 & 7.00 & 8.95 & 10.26 & 36.37 & 81.65 & 6.10 & 6.08 & 105.91 & 127.28 & 10.43 & 9.52 & 14.95 & 16.10 \\
\hline 60 & NDCor-61 & 88.00 & 92.62 & 3.07 & 6.15 & 15.69 & 11.77 & 69.86 & 64.04 & 5.70 & 8.00 & 7.95 & 8.46 & 34.87 & 55.05 & 6.20 & 6.71 & 139.00 & 137.92 & 10.14 & 10.62 & 8.52 & 9.16 \\
\hline 61 & NDCor-62 & 89.00 & 89.29 & 5.31 & 5.23 & 16.83 & 15.62 & 93.07 & 34.48 & 6.64 & 7.63 & 7.18 & 7.89 & 35.84 & 50.39 & 4.66 & 5.73 & 136.41 & 104.67 & 9.65 & 12.68 & 16.40 & 15.08 \\
\hline
\end{tabular}




\begin{tabular}{|c|c|c|c|c|c|c|c|c|c|c|c|c|c|c|c|c|c|c|c|c|c|c|c|}
\hline 62 & NDCor-63 & 87.00 & 94.29 & 4.74 & 5.89 & 16.26 & 15.40 & 89.07 & 42.98 & 6.84 & 7.03 & 9.18 & 8.49 & 37.94 & 52.59 & 5.16 & 6.51 & 134.65 & 109.26 & 9.67 & 10.91 & 17.86 & 18.36 \\
\hline 63 & NDCor-64 & 92.00 & 87.29 & 4.36 & 5.65 & 15.34 & 15.84 & 96.67 & 66.48 & 6.04 & 7.23 & 10.18 & 9.69 & 38.24 & 60.79 & 7.06 & 4.79 & 119.01 & 136.10 & 10.16 & 11.02 & 11.30 & 7.50 \\
\hline 64 & NDCor-65 & 88.00 & 90.29 & 5.15 & 5.68 & 12.98 & 13.43 & 95.67 & 51.88 & 5.44 & 7.13 & 6.78 & 9.29 & 39.44 & 74.39 & 6.16 & 6.11 & 128.96 & 132.83 & 9.43 & 11.23 & 16.38 & 17.81 \\
\hline 65 & NDCor-66 & 90.00 & 85.29 & 3.54 & 4.09 & 14.34 & 15.42 & 86.27 & 52.68 & 6.34 & 7.33 & 8.98 & 7.19 & 38.04 & 48.49 & 6.26 & 5.94 & 123.81 & 133.72 & 10.24 & 9.91 & 9.96 & 9.70 \\
\hline 66 & NDCor-67 & 89.00 & 84.29 & 4.83 & 5.24 & 15.55 & 15.24 & 72.87 & 47.08 & 7.64 & 6.83 & 7.58 & 7.69 & 38.34 & 50.19 & 6.06 & 7.02 & 124.41 & 136.63 & 9.59 & 9.98 & 15.70 & 18.50 \\
\hline 67 & NDCor-68 & 86.00 & 89.29 & 4.54 & 5.43 & 14.72 & 14.83 & 92.07 & 48.68 & 6.24 & 7.43 & 7.18 & 9.29 & 37.24 & 62.99 & 6.26 & 6.17 & 134.21 & 125.49 & 9.63 & 10.71 & 14.39 & 16.55 \\
\hline 68 & NDCor-69 & 91.00 & 88.29 & 3.14 & 4.83 & 13.74 & 14.44 & 66.87 & 55.88 & 6.14 & 8.23 & 8.58 & 6.89 & 38.94 & 38.09 & 7.46 & 5.75 & 128.19 & 140.87 & 10.15 & 9.91 & 13.43 & 17.97 \\
\hline 69 & NDCor-70 & 94.00 & 90.29 & 6.02 & 4.63 & 16.45 & 16.55 & 75.47 & 45.08 & 6.54 & 7.03 & 9.98 & 7.39 & 38.24 & 38.39 & 6.56 & 6.02 & 135.22 & 145.06 & 10.31 & 11.12 & 17.21 & 15.99 \\
\hline 70 & NDCor-71 & 88.00 & 91.29 & 3.14 & 7.50 & 12.52 & 16.04 & 59.27 & 67.38 & 6.04 & 6.03 & 8.78 & 9.19 & 39.14 & 55.99 & 5.46 & 7.43 & 128.01 & 128.51 & 11.25 & 12.25 & 12.63 & 15.08 \\
\hline 71 & $\begin{array}{l}\text { Pant Haritma } \\
\text { (Check) }\end{array}$ & 88.00 & 89.57 & 5.69 & 5.59 & 16.13 & 12.60 & 66.68 & 46.14 & 7.13 & 6.54 & 6.87 & 7.69 & 39.26 & 45.24 & 5.31 & 6.22 & 106.97 & 142.95 & 10.15 & 9.24 & 15.85 & 14.27 \\
\hline 72 & $\begin{array}{l}\begin{array}{l}\text { Hisar Anand } \\
\text { (Check) }\end{array} \\
\end{array}$ & 92.43 & 93.00 & 4.64 & 6.22 & 15.51 & 14.79 & 49.49 & 57.49 & 6.23 & 7.08 & 7.94 & 6.26 & 38.06 & 44.26 & 6.94 & 5.61 & 112.07 & 140.97 & 10.80 & 11.78 & 14.64 & 11.25 \\
\hline \multirow[t]{4}{*}{73} & $\begin{array}{l}\text { NDCor-2 } \\
\text { (Check) }\end{array}$ & 88.57 & 91.29 & 4.90 & 5.36 & 14.53 & 15.15 & 70.73 & 63.10 & 6.46 & 7.38 & 7.93 & 7.23 & 37.30 & 44.36 & 6.73 & 6.36 & 113.40 & 139.13 & 11.11 & 11.80 & 16.69 & 16.61 \\
\hline & Mean & 89.42 & 90.44 & 4.47 & 6.02 & 15.17 & 14.86 & 68.71 & 55.92 & 6.26 & 6.67 & 8.66 & 7.10 & 37.34 & 44.60 & 5.37 & 6.08 & 128.07 & 136.61 & 10.14 & 10.80 & 13.65 & 13.31 \\
\hline & Std. Dev. & 2.45 & 2.82 & 1.24 & 1.25 & 1.88 & 1.34 & 16.01 & 13.44 & 0.71 & 0.70 & 1.50 & 1.28 & 1.63 & 10.16 & 1.15 & 0.63 & 10.96 & 10.21 & 0.89 & 1.42 & 2.49 & 3.55 \\
\hline & Std. Error & 0.29 & 0.33 & 0.14 & 0.15 & 0.22 & 0.16 & 1.87 & 1.57 & 0.08 & 0.08 & 0.18 & 0.15 & 0.19 & 1.19 & 0.13 & 0.07 & 1.28 & 1.20 & 0.10 & 0.17 & 0.29 & 0.42 \\
\hline
\end{tabular}

\begin{tabular}{|c|c|c|c|c|c|c|c|c|c|c|c|c|c|c|c|c|c|c|c|c|c|c|c|}
\hline \multirow[t]{2}{*}{$\begin{array}{l}\text { S. } \\
\text { No }\end{array}$} & \multirow{2}{*}{$\begin{array}{l}\text { Characters } \\
\text { Genotypes }\end{array}$} & \multicolumn{2}{|c|}{$\begin{array}{c}\text { Days to } 50 \% \\
\text { flowering }\end{array}$} & \multicolumn{2}{|c|}{ Branches/ plant } & \multicolumn{2}{|c|}{$\begin{array}{c}\text { Fruiting nodes/ } \\
\text { plant }\end{array}$} & \multicolumn{2}{|c|}{ Umbels / plant } & \multicolumn{2}{|c|}{$\begin{array}{c}\text { Umbellets/ } \\
\text { umbel }\end{array}$} & \multicolumn{2}{|c|}{ Fruits/ umbellet } & \multicolumn{2}{|c|}{ Fruits/ umbel } & \multicolumn{2}{|c|}{$\begin{array}{l}\text { Umbel diameter } \\
\text { (cm) }\end{array}$} & \multicolumn{2}{|c|}{ Plant height (cm) } & \multicolumn{2}{|c|}{ Test weight (g) } & \multicolumn{2}{|c|}{ Yield/plant (g) } \\
\hline & & $\mathbf{Y}_{1}$ & $\mathbf{Y}_{2}$ & $\mathbf{Y}_{1}$ & $\mathbf{Y}_{2}$ & $\mathbf{Y}_{1}$ & $\mathbf{Y}_{2}$ & $\mathbf{Y}_{1}$ & $\mathbf{Y}_{2}$ & $\mathbf{Y}_{1}$ & $\mathbf{Y}_{2}$ & $\mathbf{Y}_{1}$ & $\mathbf{Y}_{2}$ & $\mathbf{Y}_{1}$ & $\mathbf{Y}_{2}$ & $\mathbf{Y}_{1}$ & $\mathbf{Y}_{2}$ & $\mathbf{Y}_{1}$ & $\mathbf{Y}_{2}$ & $\mathrm{Y}_{1}$ & $\mathbf{Y}_{2}$ & $\mathbf{Y}_{1}$ & $\mathbf{Y}_{2}$ \\
\hline & C. v. $\%$ & 2.74 & 3.12 & 20.65 & 20.76 & 12.41 & 9.03 & 23.31 & 24.04 & 11.27 & 10.43 & 17.26 & 18.01 & 14.36 & 18.78 & 15.41 & 10.33 & 8.56 & 7.48 & 8.77 & 13.12 & 18.23 & 20.66 \\
\hline & Lowest & 84.67 & 84.29 & 1.73 & 3.43 & 11.05 & 11.77 & 31.36 & 30.24 & 4.90 & 4.93 & 5.53 & 4.79 & 32.70 & 28.99 & 2.43 & 4.79 & 96.62 & 104.67 & 8.25 & 8.10 & 8.52 & 6.27 \\
\hline & Highest & 94.67 & 96.95 & 7.29 & 9.62 & 19.72 & 17.56 & 96.67 & 88.48 & 8.90 & 8.23 & 11.55 & 10.26 & 40.90 & 81.65 & 7.46 & 7.66 & 160.83 & 158.28 & 12.41 & 14.04 & 17.86 & 18.99 \\
\hline & $\begin{array}{l}\text { LSD }_{1} \\
\end{array}$ & 0.87 & 0.92 & 0.72 & 0.27 & 0.30 & 0.25 & 0.91 & 0.29 & 0.25 & 0.18 & 0.26 & 0.26 & 0.30 & 0.37 & 0.27 & 0.09 & 1.40 & 1.13 & 0.11 & 0.33 & 0.56 & 0.62 \\
\hline & $\mathbf{L S D}_{2}$ & 2.31 & 2.44 & 1.89 & 0.72 & 0.78 & 0.66 & 2.40 & 0.76 & 0.67 & 0.47 & 0.69 & 0.68 & 0.79 & 0.97 & 0.72 & 0.23 & 3.70 & 3.00 & 0.30 & 0.87 & 1.47 & 1.63 \\
\hline & $\mathrm{LSD}_{3}$ & 2.67 & 2.82 & 2.19 & 0.83 & 0.90 & 0.76 & 2.78 & 0.88 & 0.77 & 0.54 & 0.80 & 0.79 & 0.92 & 1.12 & 0.84 & 0.26 & 4.27 & 3.46 & 0.35 & 1.00 & 1.70 & 1.88 \\
\hline & $\mathrm{LSD}_{4}$ & 2.02 & 2.13 & 1.65 & 0.63 & 0.68 & 0.58 & 2.10 & 0.67 & 0.58 & 0.41 & 0.60 & 0.60 & 0.69 & 0.85 & 0.63 & 0.20 & 3.23 & 2.62 & 0.27 & 0.76 & 1.28 & 1.42 \\
\hline
\end{tabular}

Where,

$\mathrm{LSD}_{1}=$ Least significant difference between two check means.

$\mathrm{LSD}_{2}=$ Least significant difference between adjusted mean of two genotypes in same block.

$\mathrm{LSD}_{3}=$ Least significant difference between adjusted mean of two genotypes in different block.

$\mathrm{LSD}_{4}=$ Least significant difference between adjusted mean of genotype and check mean. 


\section{Fruits per umbel}

In $Y_{1}$ (2014-15) fruits per umbel ranged from 32.70 (NDCor-13) to 40.90 (NDCor-18) with the general mean of 37.34. Only two genotypes (NDCor-18 and NDCor-23) were found significantly superior over the best check Pant Haritma among seventy genotypes, for this trait. In $\mathrm{Y}_{2}$ (2015-16) fruits per umbel ranged from 28.99 (NDCor-9) to 81.65 (NDCor-60) with the general mean of 44.60. Twenty two genotypes were found significantly superior over the best check Pant Haritma (45.24) among seventy genotypes, for this trait. The top five ranked genotypes were NDCor-60, NDCor-65, NDCor-50, NDCor-68 and NDCor-64.

\section{Umbel diameter (cm)}

In $\mathrm{Y}_{1}$ (2014-15) umbel diameter ranged from 2.43 (NDCor-51) to $7.46 \mathrm{~cm}$ (NDCor-69) with the general mean of $5.37 \mathrm{~cm}$. None of the genotype was found significantly superior over the best check Hissar Anand (6.94) among seventy genotypes, for this trait. The top five genotypes having high diameter of umbel were NDCor-69, NDCor-15, NDCor59, NDCor-58 and NDCor-23. In $\mathrm{Y}_{2}$ (201516) umbel diameter ranged from 4.79 (NDCor-64) to $7.66 \mathrm{~cm}$ (NDCor-35) with the general mean of $6.08 \mathrm{~cm}$. Ten genotypes were found significantly superior over the best check NDCor-2 (6.36) among seventy genotypes, for this trait. The top five ranked genotypes were NDCor-35, NDCor-34, NDCor-16, NDCor-67 and NDCor-71.

\section{Plant height (cm)}

In $Y_{1}$ the highest and lowest value of the plant height $(\mathrm{cm})$ was observed in case of NDCor$24(160.83 \mathrm{~cm})$ and NDCor-50 $(96.62 \mathrm{~cm})$ with the general mean of $128.07 \mathrm{~cm}$. 64 genotypes were found significantly superior than the best check NDCor-2 $(113.40 \mathrm{~cm})$.
Genotypes NDCor-24 $(160.83 \mathrm{~cm})$, NDCor$10(147.36 \mathrm{~cm})$, NDCor-9 (143.46 cm), NDCor-3 (142.36 cm) and NDCor-6 (140.94 $\mathrm{cm})$ were the top five genotypes for this trait. In $Y_{2}$ the highest and lowest value of the plant height $(\mathrm{cm})$ was observed in case of NDCor$43(158.28 \mathrm{~cm})$ and NDCor-62 with the general mean of $136.61 \mathrm{~cm}$. twelve genotypes were found significantly superior than the best check Pant Haritma. Genotypes NDCor43, NDCor-5, NDCor-15, NDCor-44 and NDCor-9 were the top five genotypes for this trait.

\section{Test weight (g)}

In $Y_{1}$ (2014-15) test weight varied from 8.25 (NDCor-3) to $12.41 \mathrm{~g}$ (NDCor-34) with the general mean of $10.14 \mathrm{~g}$. Among the seventy genotypes only five genotypes viz., NDCor34, NDCor-10, NDCor-44, NDCor-6 and NDCor-9 were found significantly superior over the best check NDCor-2 for the test weight. In $Y_{2}$ (2015-16) test weight varied from 8.10 (NDCor-38) to $14.04 \mathrm{~g}$ (NDCor23 ) with the general mean of $10.80 \mathrm{~g}$. Among the seventy genotypes eight genotypes were found significantly superior over the best check NDCor-2. The top five genotypes were NDCor-23, NDCor-21, NDCor-13, NDCor12 and NDCor-26 for the test weight. Arganosa et al., (1998) in the range of 9.2-9.9 g. Maurya (1989) got 1000-fruit weight from 8.82-18.52 g while Datta and Choudhuri (2006) from 9.33-13.82 g. The result regarding 1000-seed weight of the present study was in the range of all authors except Arganosa et al., (1998)

\section{Yield per plant (g)}

In $\mathrm{Y}_{1}$ (2014-15) yield per plant ranged from $8.52 \mathrm{~g}$ (NDCor-61) to $17.86 \mathrm{~g}$ (NDCor-63) with the general mean of $13.65 \mathrm{~g}$. In the comparison of the checks seed yield per plant of seventy genotypes was found non- 
significant. However the best check was NDCor-2 (16.69 g) among the three checks for this trait. In $\mathrm{Y}_{2}(2015-16)$ yield per plant ranged from $6.27 \mathrm{~g}$ (NDCor-47) to $18.99 \mathrm{~g}$ (NDCor-39) with the general mean of 13.31 g. In the comparison of the checks seed yield per plant of seventy genotypes four genotypes produced significantly higher yield namely, NDCor-39, NDCor-53, NDCor-67 and NDCor-63. However the best check was NDCor-2 among the three checks for this trait. The present findings support the result of Bhandari and Gupta (1993). The difference in seed yield per plant might be due to the genotypic difference and ecological variation.

\section{References}

Anonymous (2016-17). Data base National Horticulture Board, Gurgaon, Hariyana

Arganosa, G. C., F. W. Sosulski and A. E. Silkard. (1998). Seed yield and essential oil of Bangladesh J. Agril. Res. 29 (1): 59-66.

Bhandari, M. M. and A. Gupta. (1993). Association analysis in coriander. Indian J. Genet.
Datta, S and P. Choudhuri. (2006). Evaluation of coriander germplasm under tetra zone of genotypes. Dept. of Hort. MS Thesis. Bangabandhu Sheikh Mujibur Rahman.

Islam, M. S., M. A. Rahman Mazumder, M. M. Hossain. R. Kundu and M. A. J. Bhuyan. J. Hort. 46 (1): 107-110.

Maurya, K. R. 1989. Growth, yield and quality component in coriander genotypes. Indian northern-grown coriander (Coriandrum sativum L). J. Herbs, Spices and Medicinal Plants 6 (2): 23-32.

Performance of some coriander genotypes for grain yield and its attributes. (2004) 53 (1): 66-70. Agricultural University (BSMRAU), Gazipur. 53 P.

Pruthi, J.S. (1998). Spices and condiments. National Book Trust, New Delhi in India. Pp.125-132.

Rahman, M. A. (2000). Morphological characters and yield potential of different coriander West Bengal. Haryana J. Hortic. Sci. 35 (3 \& 4): 348349.

\section{How to cite this article:}

Akshay Jain, V. P. Pandey, V. B. Singh, Manvendra Singh and Sriom. 2017. Evaluation of Coriander (Coriandrum Sativum L.) Genotypes for Growth and Seed Yield Attributes. Int.J.Curr.Microbiol.App.Sci. 6(8): 597-605. doi: https://doi.org/10.20546/ijcmas.2017.608.077 